\title{
Intermédialités
}

Histoire et théorie des arts, des lettres et des techniques

Intermediality

History and Theory of the Arts, Literature and Technologies

\section{L’image du trépas}

\section{Michèle Garneau}

Numéro 2, automne 2003

Raconter

Telling

URI : https://id.erudit.org/iderudit/1005461ar

DOI : https://doi.org/10.7202/1005461ar

Aller au sommaire du numéro

\section{Éditeur(s)}

Centre de recherche sur l'intermédialité

ISSN

1705-8546 (imprimé)

1920-3136 (numérique)

Découvrir la revue

Citer cet article

Garneau, M. (2003). L’image du trépas. Intermédialités / Intermediality, (2),

133-153. https://doi.org/10.7202/1005461ar

\section{Résumé de l'article}

Comment la narration de la mort d'un homme au cinéma peut-elle devenir la célébration de l'image elle-même, de sa puissance de résurrection? Cet article propose une lecture du Goût de la cerise du cinéaste iranien Abbas Kiarostami, dont la narration conduit le spectateur au seuil du visible, dans une image qui - telle est l'hypothèse ici proposée - est l'image même du spectateur que souhaite Kiarostami : celui qui, fermant les yeux sur les images qu'on lui propose, se raconterait son propre au-delà à même la finitude de l'image cinématographique. 


\title{
L'image du trépas'
}

\author{
MICHÈLE GARNEAU
}

«De nous, qui peut savoir quel bon vent nous amène et quel vent nous ramène, et à quel part? - Alors? »

Omar KHAYYÂM ${ }^{2}$

\begin{abstract}
$T$
a'm e guilass (Le goût de la cerise, 1997) du cinéaste iranien Abbas Kiarostami a une double fin: une fin narrative cinématographique et, selon l'expression du cinéaste lui-même, un «petit post-scriptum en vidéo ». Or, entre la sortie de la narration cinématographique de l'homme qui veut mourir et l'entrée dans le post-scriptum vidéographique qui se déploie sous le signe de la résurrection - sur ce seuil - le spectateur, pendant une longue minute, est plongé dans l'obscurité la plus totale. Ce qui permet le passage intermédiatique d'une fin à l'autre, c'est une image noire ${ }^{3}$, véritable figure du «seuil». Parce
\end{abstract}

1. Cette recherche est menée dans le cadre d'un projet collectif portant sur «L'intermédialité de l'expérience », et soutenue financièrement par le FQRSC. Par ailleurs, Cet article est une version longue d'une communication donnée à Udine, en Italie, en mars 2003 , dans le cadre du " $X^{e}$ Convegno Internazionale di Studi sul Cinema» intitulé: limina /le soglie del film/film's thresholds.

2. Omar Khayyâm, Sa vie et ses quatrains Rubâiyât, Pierre Seghers (éd.), Paris, Seghers, coll. «Miroir du monde», 1982, p. 12. Désormais, les références à cet ouvrage seront indiquées par le sigle «OKH», suivi de la page, et placées entre parenthèse dans le corps du texte.

3. Jean-Luc Nancy a raison de spécifier qu'il ne s'agit pas ici d'un fondu au noir, mais d'une image noire ou encore d'une image du noir. Voir L'évidence du film, Bruxelles, Yves Gevaert Éditeur, 2001, p. 79. 
qu'on peut supposer qu'en elle trépasse le personnage, cette image est une image du trépas. Mais parce qu'elle fait passer le spectateur dans une autre image, elle est une image trespas, au sens étymologique latin de "passage », une image qui fait passer. En faisant passer dans une autre image, l'image est devenue autre, et c'est à ce devenir altérant, à cette altérité de l'image que je m’intéresserai tout particulièrement.

Qu'est-ce qu'un seuil? «C'est un espace définissable comme bref, délimitant deux lieux et procédant l'un de l'autre; à la fois entrée et sortie. Il impose à celui qui le franchit une démarche, un pas. " L'idée de franchissement liée à la notion de seuil, ne doit pas occulter l'idée d'arrêt ou encore d'hésitation (sur un seuil). Giorgio Agamben s'est penché sur ces deux puissances de l'image que sont la puissance de répétition et la puissance d'arrêt et lie cette dernière à la césure poétique. Si la césure poétique est un arrêt, nous dit-il, « ce n'est pas au sens d'une pause, chronologique, mais plutôt au sens d'une puissance d'arrêt qui travaille l'image elle-même, qui la soustrait au pouvoir narratif pour l'exposer en tant que telle ${ }^{5} »$. On pourrait dès lors concevoir cette image noire en tant que césure poétique. Et ce qui hésiterait ici, sur ce seuil, c'est le sens à donner à l'image, à l'image elle-même autant qu'à ce qui lui succède. Agamben reprend la définition que Paul Valéry donne du poème - Le poème, une hésitation prolongée entre le son et le sens - et l'adapte, pour les besoins de sa démonstration, au cinéma: «On pourrait [...] dire du cinéma, du moins d’un certain cinéma, qu'il est "une hésitation prolongée entre l’image et le sens". " En hésitant ainsi sur le seuil qu'elle figure, l'image interpelle son spectateur. Or, si cette image est une image-seuil, ce n'est pas seulement parce que nous sommes suspendus, pendant toute sa durée, entre la vie et la mort, entre la mort et la résurrection, entre la nuit et le jour, entre le ciel et la terre, entre l'obscurité et la lumière, mais aussi parce que cette image impose à celui qui la regarde une démarche qui est celle-là même de l'interprétation. Le petit postscriptum vidéographique qui succède à l'image noire (mais en procède) place le spectateur à l'intérieur d'une double irrésolution: celle ressortissant au dénouement cinématographique de la narration (le héros est-il mort, toujours

4. Dominique Arban, «Le "seuil" - thème, motif et concept», Cahiers de l'Herne, n 24, «Dostoïevski », 1973, p. 205.

5. Giorgio Agamben, «Le cinéma de Guy Debord», Trafic, n²2, été 1997, p. 59.

6. Giorgio Agamben, «Le cinéma de Guy Debord», p. 59. 
vivant ou ressuscité?) et celle ressortissant au dénouement vidéographique de la méta-narration (le film est-il sauvé par cette réflexivité qui fait retour sur le caractère fictif de la narration?). Or, la démarche interprétative que je propose n’implique nullement que nous choisissions entre la vie, la mort ou la résurrection du héros - bien qu'il nous soit tout à fait loisible de faire ce pas, un pas qui viendrait boucler le sens, clore la représentation.

Il s'agira plutôt d'une démarche qui implique que nous entrions dans un jeu, «[...] mais pour que le jeu s'accomplisse, prévient Roland Barthes, il faut respecter certaines règles; il faut d'une part que l'œuvre soit vraiment une forme, qu'elle désigne vraiment un sens tremblé, et non un sens fermé $[\ldots]^{7}$ » Ce dont nous parle ici Barthes, se préparant à répondre à Racine, c'est de la collaboration théorique, mentale, du lecteur qui doit interpréter librement un fait esthétique doué d'une structure donnée (même si cette structure doit permettre une infinité d'interprétations). Or, cette infinité d'interprétations, c'est la centaine de films que souhaite Kiarostami : «Je crois à un cinéma qui donne plus de possibilités et de temps à son spectateur. Un cinéma mi-fabriqué, un cinéma inachevé qui se complète avec l'esprit du spectateur et, du coup, nous avons une centaine de films 8 . » Dans tous ses entretiens, l'énigmatique cinéaste ne cesse d'insister sur la collaboration du spectateur. "Je préfère offrir plusieurs interprétations possibles, pour laisser le spectateur libre de choisir. » Il ne s'agit pas ici de satisfaire à l'arborescence du récit interactif. Sous l'apparente banalité de la déclaration se cache une pratique du cinéma qu'il convient de replacer dans son contexte de production, un contexte de haute surveillance de l'image qui fait resurgir la question du sens. Cinéaste de l'ambiguïté, Kiarostami ne veut que faire osciller le sens, le soumettre à un salutaire tremblement, afin de l'empêcher d'être jamais assuré.

Dans l'image noire, nous sommes suspendus - et ce sera là ma démonstration dans la première partie - à deux interprétations qui, ironie persane oblige, se retournent en deux interprétations de l'interprétation. Entre la néces-

7. Roland Barthes, Sur Racine, Paris, Éditions du Seuil, 1963, p. 11.

8. Abbas Kiarostami, «Un film, cent rêves», dans Abbas Kiarostami. Textes, entretiens, filmographie complète, Paris, Éditions de l'Étoile/Cahiers du cinéma, 1997, p. 71. Désormais, les références à cet ouvrage seront indiquées par le sigle «AK», suivi de la page, et placées entre parenthèse dans le corps du texte.

9. Thierry Jousse et Serge Toubiana, «Un film n'a pas de passeport..., Entretien avec Abbas Kiarostami », Paris, Cahiers du cinéma, n 541, décembre 1999, p. 30. 
sité de chercher un sens - pour jouer - et la vérité du sens comme présence pleine, fondement rassurant, il y a «les deux interprétations de l'interprétation qui, selon Jacques Derrida, se partagent le champ de ce qu'on appelle les sciences humaines ${ }^{10}$ ». Au concept de jeu de la première, il faut opposer les concepts d'être et de vérité de la deuxième. Or, ces deux interprétations de l'interprétation «sont inconciliables, affirme Derrida, et ce, même si nous les vivons simultanément et les concilions dans une obscure économie ${ }^{11}$ ». Il s'agira de voir comment, dans ce film, et pour quelles raisons, ces deux interprétations se concilient sans pour autant se réconcilier. En deuxième partie, j’examinerai les images du post-scriptum. Après avoir «introduit dans la vue », par une image noire, «ce retournement qu'est l'extase et qu'est la mort ${ }^{12}$ », Kiarostami projette le spectateur dans une image des plus ambiguës, sorte de stase de désorientation. Ce basculement dans une autre image a beaucoup désarçonné la critique. On a parlé de «déchet dérisoire et un peu grotesque», de «nouvelle aube ou lendemain d'apocalypse ${ }^{13}$ ?»; ou encore «d'obligation postmoderne de célébration », d' «idée mystique ancienne de la nécessaire rédemption de tout ${ }^{14}$ ». Que raconte le post-scriptum, s'il raconte encore quelque chose? Telle est la question que nous poserons en guise de conclusion.

Commençons d'abord par proposer deux interprétations de l'image noire. Nous hésiterons ici entre l'image de la résurrection et la résurrection par l'image, entre une image de la Toute-Puissante - en tant qu'elle annonce la Résurrection - et la puissance de l'image - en tant que «forme de résurrection ${ }^{15}$ ». Est-ce un hasard si, dans un film iranien, nous sommes en présence d'un des motifs les plus profonds du cinéma: la résurrection, ou encore, l'éternelle

10. Jacques Derrida, L'écriture et la différence, Paris, Éditions du Seuil, 1967, p. 427.

11. Jacques Derrida, L'écriture et la différence, p. 427.

12. Maurice Blanchot, L'espace littéraire, Paris, Éditions Gallimard, coll. «Idées NRF", 1955, p. 198. La phrase complète se lit comme suit: «Voir comme il faut, c'est essentiellement mourir, c'est introduire dans la vue ce retournement qu'est l'extase et qu'est la mort. »

13. Emmanuel Burdeau, «Le goût de la cerise d'Abbas Kiarostami », Paris, Cahiers du cinéma, $\mathrm{n}^{\circ} 518$, novembre 1997, p. 65.

14. Youssef Ishagpour, Le réel, face ou pile. Le cinéma d'Abbas Kiarostami, Tours, Farrago, 2000, p. 29.

15. Abbas Kiarostami, "À propos du Goût de la cerise, Entretien», dans Abbas Kiarostami. Textes, entretiens, filmographie complète, p. 81. 
naissance du monde, et que ce thème soit d'abord un thème religieux et politique? Voici quelques versets du Coran:

C'est Dieu qui déchaîne les vents comme une annonce de sa miséricorde.

Lorsqu'ils portent de lourds nuages, nous les poussons vers une terre morte; nous en faisons tomber l'eau avec laquelle nous faisons croître toutes sortes de fruits.

Nous ferons ainsi surgir les morts.

Peut-être réfléchirez-vous? (VII, $57^{16}$ )

C'est Dieu qui déchaîne les vents; ceux-ci soulèvent les nuages que nous poussons vers une terre morte.

Nous rendons ainsi la vie à la terre après sa mort.

Voilà comment se fera la Résurrection (XXXV, 9 $\left.9^{17}\right)$

Dans le Coran, comme dans les images du film, la puissance de la Résurrection se manifeste en signes visibles, signes divins de la pluie et du vent. Les effets de la pluie, bienfait de Dieu, sont considérés par le Coran comme un signe, une preuve de la Résurrection des morts. Le vent est l'intermédiaire entre le monde terrestre et les puissances surnaturelles dont il traduit l'action en termes matériels. Du fond de sa fosse, M. Baadi regarde les lourds nuages poussés par le vent. Après que son visage eut été purifié par l'eau de la pluie, il ressuscite dans un monde où les soldats sourient et cueillent des fleurs. Le vent, dans le post-scriptum, est d'ailleurs ce que l'équipe du tournage est venue enregistrer. Ainsi est-il possible de lire dans ces images une transposition du Coran, de les voir avec les yeux de la Foi. Dans un entretien, le cinéaste a d'ailleurs déclaré :

Committing suicide is forbidden in Islam, of course, and is not even spoken of. But some religious people have liked the film because they felt that, just as you said, it shows a quest to connect with some thing more heavenly, something above physical life. The scene at the end, where you see cherry blooms and beautiful

16. Le Koran, trad. Claude Savary [1782-1783], Paris, Éditions Garnier Frères, 1960, p. 216 .

17. Le Koran, p. 423. 
things after he's died, has that message-that has opened the door to heaven. It wasn't a hellish thing he did, it was a heavenly transition ${ }^{18}$.

Comment, dans cette perspective, regarde-t-on le post-scriptum? Comme l'image même de la résurrection. Cette interprétation, en tant que fin narrative, rédemptrice en Dieu, a tout pour rassurer les mollahs-censeurs. La mort s'accomplit sous la bienveillance divine. Son sens est garanti par le Livre suprême. C'est une image licite ayant son fondement dans la religion.

Voici maintenant quelques vers d'Omar Khayyâm, ce poète et scientifique persan du XII ${ }^{\mathrm{e}}$ siècle que le cinéaste a souvent évoqué dans ses entrevues avec la presse internationale ${ }^{19}$.

Nous sommes tous pions aux mains du Ciel. Pour se distraire,

Il nous déplace

Ce n'est pas ici métaphore, il est le Maître, seul il joue

Sur l'échiquier de l'existence il nous pousse sans nous connaître

Puis il nous jette un par un par un dans l'étroit cercueil du Néant. (OKH, p. 77)

Nous ne sommes que des marionnettes et le ciel est notre montreur

Ce n'est pas ici métaphore

Sur l'Échiquier de l'existence, nous sommes voués à nos jeux

pour tomber, eux finis, dans le néant d'un coffre. (ОКн, p. 25)

Ici, c'est le Ciel qui est le Maître. Dans le jeu cosmique que l'univers joue avec lui-même, l'homme n'est qu'un élément parmi d'autres. Sans le connaître, et sans que nous puissions le connaître, Kiarostami jette son personnage dans

18. David Sterrit, «Taste of Kiarostami», Senses of Cinema, 2000, http://www. sensesofcinema.com/contents/oo/g/kiarostami.html. Il s'agit d'une version courte d'un entretien paru dans Film Comment, vol. 36, nº 4, juillet-août 2000.

19. «Khayyâm a une place si importante en Iran qu'il est intouchable. [...] Dans mes films, la référence à Khayyâm date de Et la vie continue lorsque je suis allé sur les lieux du tremblement de terre pour constater le désastre. Avant cela, je n’avais jamais vu d'aussi près la contradiction entre la vie et la mort. À ce moment-là, j'ai mieux saisi la profondeur de la poésie et de la philosophie de Khayyâm. Elle repose sur ce principe: pour admirer la vie, il faut approcher de très près la mort, la regarder dans les yeux. » (Thierry Jousse et Serge Toubiana, «Un film n'a pas de passeport...», p. 31) Pierre Seghers, dans sa présentation des Rubâ’iyât (quatrains) de Khayyâm, écrit: "Contre l'hypocrisie des cœurs éteints, ceux des mollahs, il se laisse aller à une audace qui pouvait coûter cher en ce temps-là. Aujourd'hui aussi, il est vrai: dans le vieil Iran, rien ne change. » (Omar Khayyâm, Sa vie et ses quatrains Rubâ'iyât, p. 15) 
l'étroit cercueil du néant. Puis, pour nous distraire - de ce néant - , il déplacera la narration cinématographique vers la célébration vidéographique. L'image noire ne serait donc plus ici la mort accomplie, ou la mort qui s'accomplit sous la bienveillance divine, mais «la matérialisation de la mort, du néant» (AK, p. 81).

Citons alors un autre quatrain de Khayyâm:

Jusqu'à quand iras-tu, réfléchissant sur ta Substance ou bien t'interrogeant sur l'Être et le Néant?

Bois du vin! La mort talonne cette vie

Préfère le sommeil ou l'ivresse aux questions. (OKH, p. 68)

«Les quatrains de ce poète, nous dit Kiarostami, sont un constant éloge de la vie, avec une omniprésence de la mort. La mort lui sert à saisir la vie.» (AK, p. 8o) Il y a chez Khayyâm une affirmation joyeuse du jeu du monde, l'affirmation d'un monde de signes sans fautes, sans vérité, sans origine. Khayyâm est un épicurien, note Seghers. «Il a tout appris des Grecs à travers son maître Bahmanyâr, lui-même disciple d'Avicenne, et qui suivra, comme Lucrèce, les leçons d'Épicure. » (OKH, p. 26) Contrairement à la fiction de la fin que propose le Coran, en tant que modèle du monde, il n'y a pas, ici, de consolation métaphysique.

On pourrait encore appréhender l'image noire à la manière de la philosophie occidentale, comme pur dehors. Le dehors en tant qu'il fait surgir ce qu'il y a d'impensé dans la pensée. Un homme quelconque est livré à ce que Maurice Blanchot appelle la «nuit ${ }^{20} »$. La nuit et le dehors dont elle témoigne, dehors entendu ici comme hors monde et hors être. L'image noire nous renverrait au neutre et non à l'être - à la neutralité absolue de la mort. Mais comme le film ne se termine pas sur cette image noire qui, en quelque sorte, est relancée par les images du post-scriptum, il convient d'aller plus avant dans cette lecture qui prend en compte le concept de dehors blanchotien. Ni Dieu, ni Néant, devrions-nous dire, car, dans les deux cas, c'est la clôture de la représentation. Rendre justice, ni à la mort, ni à Dieu, mais à la vie en ce qu'elle connaît la mort. Car s'il faut, dans l'optique blanchotienne, préserver la mort de toute interprétation rédemptrice, il convient aussi de ne pas se laisser fasciner par elle. Entre la présence de la mort en train de s'accomplir sous la bienveillance divine et celle des pouvoirs de fascination de la Nuit, il y aurait 
quelque chose comme la «liberté de la Nuit» qui, chez Blanchot, est de l'ordre d'un acte de purification.

L'acte de la Nuit, ce serait le mouvement par lequel M. Baadi «purifie sa mort en lui ôtant le caractère d'un hasard ${ }^{21}$ ». Monsieur Baadi veut choisir «sa » mort contre la mort anonyme, contre le hasard, ou contre la volonté divine. «Il y a dans la décision de se tuer, une forme de liberté, avance Kiarostami : c'est le seul moment où nous pouvons tenir tête à Dieu ou à la Nature. » (AK, p. 75) L'omniprésence des soldats tout au long du film me permet d'avancer cette hypothèse. «L'angoisse de la mort anonyme, l'angoisse du On meurt, et l'espoir du je meurs, où se retranche l'individualisme ${ }^{22}$ » inviterait le personnage à vouloir donner son nom et son visage à l'instant de mourir. Car M. Baadi ne veut pas seulement mourir, il veut être vu mort. Il demande qu'on l'appelle par son nom, qu'on se penche sur sa fosse pour vérifier s'il est bien mort. Monsieur Baadi veut avoir sa mort et être nommé, salué par cette mort unique. Le sens de son identité doit l'emporter sur la soumission au destin collectif. «Tout était parfait, écrit encore Blanchot, elle était la Nuit et elle entendit son propre cœur qui battit ${ }^{23}$. " Ce que l'image noire ferait entendre, car cette image aveugle n'est pas sourde - avant que le silence ne se fasse pendant quelques secondes, on y entend le bruit de la pluie - serait la purification de la Nuit. «Non pas Dieu dans sa transcendance, comme l'a fort justement écrit Ishagpour, mais un renversement négatif dans la terre et dans la tombe, dans la nuit et sous la pluie ${ }^{24}$. »

La mort que nous propose Kiarostami par le biais de son personnage n'en est pas moins scandaleuse. Non seulement M. Baadi veut-il se suicider, mais il veut se faire enterrer en transgressant les rites d'enterrement religieux de sa société. La mort que revendique le personnage est une mort «a-symbolique, hors religieux, hors rituel, sorte de plongée brusque dans la mort littérale ${ }^{25}$ ». C'est la «Mort plate», contemporaine du recul des rites, que Roland Barthes associait à la photographie ${ }^{26}$.

21. Maurice Blanchot, L'espace littéraire, p. 204.

22. Maurice Blanchot, L'espace littéraire, p. 163.

23. Maurice Blanchot, L'espace littéraire, p. 144.

24. Youssef Ishagpour, Le réel, face ou pile, p. 91.

25. Roland Barthes, La chambre claire. Note sur la photographie, Paris, Cahiers du cinéma, Éditions Gallimard, Éditions du Seuil, 1980, p. 144.

26. «Avec la photographie, nous entrons dans la Mort plate. » (Roland Barthes, La chambre claire, p. 145) 
La société iranienne, à travers son cinéma, serait-elle en train d'accueillir une telle conception de la mort? Une mort narrée «à même la finitude de l'image ${ }^{27} »$ ? Qu'une telle mort soit revendiquée par l'image photographique n'est peut-être pas un hasard. Nous serions dès lors non plus dans une fin au sens de dénouement poétique, dans une «image de la résurrection », mais dans une fin au sens philosophique de finitude, et vécue entièrement à même la finitude de l'image.

Si donc il importe de ne pas occulter la nuit, s'il faut lui rendre justice, sauvegarder sa donation sauvage, la pensée, face à son mystère, ne peut qu'abdiquer, admettre sa finitude. En exhibant ainsi, pendant quelques secondes, une image totalement noire et silencieuse, Kiarostami suggérerait-il que nous sommes arrivés, non pas dans l'au-delà, mais au-delà d'une possibilité de sémiotisation, qu'il n'y a plus rien à voir? Je dirais plutôt que le cinéaste s'arrête, hésite auprès (au seuil) de l'énigme, entendue comme ce qui est irréductible à tout dévoilement. La mort, écrit Nancy, «reste secrète, incompréhensible, inappropriable ${ }^{28} »$. L'image garde son secret, ou une réserve essentielle.

Derrière ce rideau, personne n'a l'accès

Personne ne pénètre et perce le mystère

Notre seule maison est au sein de la terre

Ô regrets! Une énigme, une plaie, un secret (окн, р. 57)

Comment, dans cette deuxième perspective interprétative, regarde-t-on le post-scriptum? S'il n'y a pas de signifié transcendantal, s'il n'y a ni Dieu, ni Néant, ni Être, ni Non-Être qui, en tant que centre, arrête et fonde le jeu des substitutions, on peut dès lors étendre à l'infini le champ et la signification des images. On peut dès lors entrer dans le mouvement du jeu «dont on efface toujours en français la signification scandaleuse». Ce mouvement du jeu, poursuit Derrida, «permis par le manque, l'absence de centre ou d'origine, est le mouvement de la supplémentarité29 ». Ce qui empêche le signe de se fermer sur soi, en le rappelant à sa différence, c'est ce que Derrida, on le sait, a appelé

27. Je reprends l'expression à Ishagpour: "C'est une mort que rien ne permet de transformer: sa vérité, c'est l' “absence d'œuvre”, vécue entièrement à même la finitude de l'image.» (D'une image à l'autre, la nouvelle modernité du cinéma, Paris, Éditions Denoël/Gonthier, 1982, p. 163)

28. Jean-Luc Nancy, L'évidence du film, p. 37.

29. Jacques Derrida, L'écriture et la différence, p. 423. 
le mouvement de la supplémentarité. Ce sont les quatrains de Khayyâm, qui ne cessent de proliférer autour de l'absence de sens de tout, mais c'est aussi bien le post-scriptum qui prolifère sur lui-même en faisant signe d'un passage, que le «vent nous emportera », ou que «la vie continue ». "C'est un document que l'on a annexé au film, nous dit Kiarostami. Je ne veux pas que cette partie soit partie intégrante du film.» (AK, p. 85) Le post-scriptum vidéographique est un supplément au film, mais il est aussi ce qui vient supplémenter le manque qu'on assume dans la légèreté de la nouvelle image.

Les deux interprétations de l'image noire proposées ici nous renvoient aux deux interprétations de l'interprétation:

L'une cherche à déchiffrer une vérité ou une origine échappant au jeu et à l'ordre du signe; l'autre affirme le jeu et tente de passer au-delà de l'homme et de l'humanisme, le nom de l'homme étant le nom de cet être qui, à travers l'histoire de la métaphysique et de l'onto-théologie, c'est-à-dire du tout de son histoire, a rêvé de la présence pleine, le fondement rassurant ${ }^{30}$.

D’un côté, une image de la vérité, de l'autre, la vérité de l'image? Une image de la vérité, une image véridique, qu'est-ce que c'est? C'est une image transcendante, une image dans laquelle la vérité ne se construit pas, mais se reconnaît. L'image est le lieu d'une transcendance quand on lui accorde le pouvoir de manifester une puissance supérieure, extérieure à elle et la surplombant. Quelle serait maintenant l'autre vérité de l'image noire, à savoir, la vérité de l'image?

La conception deleuzienne de l'image-temps, qui s'appuie sur l'idée du dehors blanchotien, fournit des éléments de réponse. Ce que Deleuze appelle le dehors correspond à la définition de Blanchot: c'est l'impensé dans la pensée, ou encore, le dehors de la représentation, le non-représentable. En suspendant la narration pour s'exposer en tant que telle, dans sa puissance d'arrêt, l'image noire forcerait la pensée à atteindre son dehors. La regarder, ce serait «se mettre à l'épreuve d'un sens qu'on ne maîtrise pas $^{31}$ ». Et il n'y aurait rien à reconnaître dans l'image noire, rien à retrouver; pas de postulat de la recognition. C'est pourquoi l'image de la Toute-Puissance, image de la transcendance, peut-elle devenir, dans la deuxième lecture que j'en propose, une puissance du dehors,

30. Jacques Derrida, L'écriture et la différence, p. 423.

31. Jean-Luc Nancy, L'évidence du film, p. 39. 
une «situation optique et sonore pure ${ }^{32}$ ». Le post-scriptum ne serait pas une image véridique (en tant qu'image de la résurrection), mais la vérité de l'image quand sa force est dans cette «équivoque » dont nous parle Derrida, une «équivoque pure et infinie ne laissant aucun répit, aucun repos au sens signifié, l'engageant, en sa propre économie, à faire signe encore à différer... ${ }^{33}$ » Quelle est la différence entre le signe laïc et le signe religieux? Peut-être n'y en a-t-il aucune. Peut-être ne s'agit-il ici que de lutter contre l'intégrisme, c'est-à-dire l'appartenance à un seul signe. «L'art permet à l'individu de créer sa vérité selon ses souhaits et ses critères, a déclaré Kiarostami ; il permet aussi de ne pas accepter d'autres vérités imposées.» (AK, p. 70) Si l'image noire fait signe vers un au-delà, il nous est loisible de choisir entre un au-delà au sens religieux l’image de la Résurrection - et un au-delà médiatique - , une résurrection de l'image.

L'ambivalence de l'image chez Kiarostami nous permet d'envisager l'inclusion du dehors dans le monde, et non plus seulement au-delà, outre-monde. Le dehors, que ce soit chez Blanchot, Derrida, Foucault ou Deleuze, est cette catégorie immanente, condition d'une pensée de l'immanence radicale. Dans l'image-temps, figure de l'immanence chez Deleuze, le dehors ou l'envers des images ont remplacé le Tout. «Le tout se confond alors avec ce que Blanchot appelle la force de "dispersion du Dehors" ou le "vertige de l'espacement" [...] : ce vide qui n'est plus une part motrice de l'image, et qu'elle franchirait pour continuer, mais qui est la mise en question radicale de l'image ${ }^{34}$. " Si le statut de l'image noire, toujours dans la terminologie deleuzienne, peut être dit «intersticiel», c'est dans la mesure où cette image nous sort d'une chaîne d'images, de l'enchaînement narratif qui nous liait au regard de l'homme qui veut mourir. La chaîne associative et causale des images sur le monde d'un homme en proie à la maladie de la mort s'interrompt, et nous nous retrouvons devant un peu de temps à l'état pur. L'image noire qui s'expose ici en tant que telle, tout en faisant trépasser le personnage et tout en le faisant passer dans un autre monde, ferait aussi passer du temps. En elle - ou à travers elle - un ange passe...

32. C'est la définition même de l'image-temps. Voir tout le premier chapitre de Cinéma 2. L'image-temps, Paris, Éditions de Minuit, 1985.

33. Jacques Derrida, L'écriture et la différence, p. 42.

34. Gilles Deleuze, Cinéma 2. L'image-temps, p. 235. 
La deuxième fin du Goût de la cerise a beaucoup désarçonné la critique. Essayons de décrire de qui s'y passe afin de dégager ce que j'ai appelé l'altérité de cette image. «Si j’ai tourné cette partie finale en vidéo, a déclaré Kiarostami, c'est pour ne pas être contraint de poursuivre cette histoire - de décider si l'homme était vivant ou non. Pour moi, cela n'est pas important. Je suppose qu'en principe, il aurait dû mourir. Mais il a peut-être été sauvé le lendemain matin. » (AK, p. 81) Nous aurions ici une autre finalité du post-scriptum: une fonction de libération de la narration. Par cette image noire, Kiarostami nous sort de l'enchaînement narratif, mais non de la fiction. «Je n'ai pas voulu forcer l'interprétation du spectateur, mais tenté de faire comprendre que ce qu'il avait vu jusque-là était une narration.» (AK, p. 81) Ne confondons pas ici, «fin de l'action imitée et fin de la fiction en tant que telle ${ }^{35} »$. En début d'article j’ai parlé d'irrésolution touchant le dénouement cinématographique de la narration (le héros est-il mort, toujours vivant ou ressuscité?) et le dénouement vidéographique de la méta-narration (le film est-il sauvé par cette réflexivité qui fait retour sur le caractère fictif de la narration?).

La terminaison du film est non conclusive, et ce, dans sa première comme dans sa deuxième fin. Kiarostami, on l'a vu, s'arrête au seuil de l'énigme. Son image est une énigme, elle est l'énigme même de la mort. Dans le post-scriptum, on le verra dans un instant, le film se termine en faisant signe vers un recommencement, la promesse d'une suite. «Une terminaison non conclusive convient à une œuvre qui soulève à dessein un problème que l'auteur tient pour insoluble, écrit Ricœur. L'inconclusion déclare en quelque sorte l'irrésolution du problème posé3 ${ }^{36}$. Or, si l'inconclusion déclare l'irrésolution du problème posé, pourquoi faire retour sur le caractère fictif du film? Pourquoi faut-il insister sur le caractère fictif de l'énoncé cinématographique en filmant une scène de tournage du film? Pour répondre à cette question, il faut revenir au contexte de production. Le danger qu'avec le cinéma la religion perde son statut de vérité ultime, de fondement de toutes les images est présent en Iran. Car fiction et religion sont très proches. Thomas Pavel l'a bien montré dans son ouvrage sur la fiction: les mondes sacrés et fictionnels tissent le même genre de liens allégoriques entre deux niveaux différents: une réalité qui se caractérise

35. Paul Ricœur fait cette distinction importante dans Temps et récit II. La configuration dans le récit de fiction, Paris, Éditions du Seuil, coll. «L'ordre philosophique », 1984 , p. 38 .

36. Paul Ricœur, Temps et récit II, p. $3^{8 .}$ 
par la pauvreté et l'instabilité ontologique, et une réalité fictionnalisée ou mythifiée qui, se déployant dans un espace privilégié, jouit, elle, de la plénitude ontologique. L'espace-temps de cette réalité détient plus de poids et de stabilité que l'espace des mortels.

Comme dans l'univers de la fiction, la mentalité religieuse divise l'univers en deux régions qualitativement différentes. L'espace est partagé en régions sacrées, dotées de réalité dans le sens le plus fort du terme, et endroits non sacrés instables; le temps sacré cyclique contraste avec la durée profane irréversible ${ }^{37}$.

L'idée centrale de Pavel est que le monde de la fiction jouit de la même plénitude ontologique que le monde sacré religieux. Insister sur le caractère fictif des images filmiques, c'est insister sur le fait qu'elles n'ont pas, comme celles de la religion, un statut de vérité. C'est suggérer qu'elles ne feront pas de concurrence déloyale aux images primordiales, c'est-à-dire à celles du monde du mythe religieux.

Ishagpour rappelle le prestige du cinéma en Iran et le désir universel d'avoir son image, de devenir visible à tous. Kiarostami, quant à lui, explique dans une entrevue: "Je ne peux pas vous décrire ce phénomène de fascination par l'image en Iran. Il faudrait que vous puissiez le voir de vos propres yeux. Parfois, les gens sortent à trois heures du matin des salles de cinéma. Parfois, ils attendent des heures pour obtenir un ticket d'entrée dans une salle.» (AK, p. 50) Peut-être pourrions-nous partir d'un soupçon, le soupçon que les fictions cinématographiques, dans l'Iran actuel, puissent usurper l'autorité des Récits fondateurs à teneur religieuse qui font la Loi de la communauté. Cette question est abordée par Paul Ricœur dans son exploration du rapport entre fiction et mythe brisé. Ricœur parle de la «fonction de suppléance exercée par la fiction littéraire à l'égard des autres Récits qui ont fait autorité dans le passé de notre culture ${ }^{38}$ ». Dans Nema-ye Nazdik (Close up, 1990), Abbas Kiarostami s'intéresse à l'histoire vécue d'un pauvre homme qui a usurpé l'identité de son cinéaste préféré, Moshen Makhmalbaf. L'homme en question, Sabzian, déclare même préférer la lecture du Cycliste ${ }^{39}$ à celle du Coran. La rivalité entre ces deux formes d'autorité que sont la justice divine et la justice cinématographique est admirablement mise en scène dans le film au moment du procès. Le close up est une

37. Thomas Pavel, L'univers de la fiction, Paris, Éditions du Seuil, 1986, p. 80.

38. Paul Ricœur, Temps et récit II, p. 45.

39. Bicycleran (Le cycliste, 1987) est un film de Moshen Makhmalbaf. 
autre justice, une autre manière de rendre justice. C'est par le close up cinématographique que l'homme retrouvera sa dignité et son identité.

Le soupçon dont il est ici question, c'est le soupçon que le cinéma dise plus vrai que la religion, qu'il puisse devenir un mode de révélation qui consolerait davantage que celui de la Révélation religieuse; c'est le soupçon que le cinéma puisse devenir une religion de la survie, du salut, qu'il puisse atteindre un statut qui dépasse celui de simple divertissement. Cette question concerne le régime éthique des images. Dans ce régime, nous dit Jacques Rancière,

«l'art» n'est pas identifié tel quel, mais se trouve subsumé sous la question des images. Il y a un type d'êtres, les images, qui est l'objet d'une double question, celle de leur origine et, en conséquent, de leur teneur en vérité, celle de leur destination: des usages auxquels elles servent et des effets qu'elles induisent. Relève de ce régime la question des images de la divinité, du droit ou de l'interdiction d'en produire, du statut et de la signification de celles que l'on produit ${ }^{40}$.

Ces questions que nous ne nous posons plus en Occident, les cinéastes iraniens doivent les affronter. La question est cruciale dans une culture où fiction et religion entrent en rivalité.

Faire voir le dispositif dans le cinéma iranien, ce serait faire voir l'origine et la destination des images. Ce serait, en quelque sorte, les laïciser. L'omniprésence du dispositif cinématographique dans les titres mêmes de Salaam cinéma, réalisé par Moshen Malhalbaf en 1995, et de Close up, est révélateur. Mais, plus fondamentalement encore, il importe d'exposer l'opération par laquelle une communauté est là, présente à elle-même, manifestant un autre désir de communauté que celui qui est pris en charge, médiatisé par le dispositif religieux. On pourrait avancer l'hypothèse que c'est peut-être le cinéma qui assume actuellement en Iran la tâche de médiatiser et de narrer l'expérience singulière pour la faire entrer dans le domaine public. Cette narration, toutefois, doit être ramenée au geste d'une performance, à la célébration de l'artifice, à l'idée que l'image se fabrique et que, par la médiation cinématographique, nous ne sommes plus dans un processus d'identification, mais de fabulation. Car, dans le cinéma iranien, le peuple est là, il ne «manque pas $^{41}$ ». Les caméras de Salaam

40. Jacques Rancière, Le partage du sensible. Esthétique et politique, Paris, Éditions la Fabrique, 2000, p. 27.

41. L'expression est de Gilles Deleuze, parlant du peuple dans le cinéma moderne. Voir le chapitre "Cinéma, corps et cerveau, pensée», dans L'image-temps, p. 281-291. 
cinéma de Moshen Makhmalbaf ont peine à le contenir. Dans ce film - qui est tout autant un salut au peuple iranien qu'au cinéma - nous assistons à une (auto)mise-en-scène d'un cinéaste et de son peuple, où l'improvisation, la surprise, les aléas du tournage côtoient la répétition, l'artifice et la duperie (surtout du côté du spectateur). Une véritable communauté appareillée se met en scène, le cinéaste et ses dispositifs ayant une part égale aux sujets qui sont filmés. Les interrogations sur les modes de présence de l'image comme simulacre et son apport à la réalité sont au centre du film.

Si la machine est là, dans Salaam cinéma, s'exposant comme telle, à la fois dans ce qu'elle peut avoir de tyrannique comme de libératoire et de révélatrice, ce n'est pas pour récuser le sujet, mais pour le pousser au vertige. Le détour par la machine revient à mettre en jeu toutes sortes de débordements subjectifs. Ce nouage du sujet à la machine est hautement subversif puisqu'il présuppose que le sujet est peut-être moins à l'image de Dieu qu'une image qui se construit par la machine. Le nouge du sujet à la machine, c'est d'abord la négation de la neutralité de l'appareil de prise de vue, l'affirmation de sa relation à la société globale. Si on filme le dispositif cinématographique - des caméras filmant des caméras - c’est précisément, et contrairement au dispositif religieux, pour qu'il ne soit pas perçu comme quelque chose d'indépendant et de tout-puissant, pour que le sujet ne reçoive pas comme implacable et naturel ce qui est artificiel, construit par ses propres dispositifs. Enfin, pour qu'il ne s'ignore pas créateur, source de ses images. Le potentiel insurrectionnel du dispositif cinématographique, objet de croyance et de vénération, dans la reconfiguration de l'expérience iranienne et de sa modernisation, a souvent été souligné. À la médiation religieuse de l'homme pensé «à l'image» de Dieu, la médiation audiovisuelle oppose moins des images de l'homme qu'un dispositif permettant à celui-ci d'affronter ses propres images, son propre imaginaire.

Tournons-nous maintenant vers la fin de la fiction, c'est-à-dire le postscriptum. Si la distanciation ironique du post-scriptum libère le cinéaste de son emprise sur la narration - ou de l'emprise de la narration - nous n'en sommes pas moins encore dans l'image. C'est une image autre. Mais qu'est-ce qui est autre ici, et comment l'image construit-elle cette altérité? La mort ne se résout pas dramatiquement, mais dans l'image, par l'image, par la différenciation de deux types d'images. L'image s'est désincarnée. Parlant de l'imagevidéo, Pascal Bonitzer écrit: «Les corps sont libérés de toutes les émotions, de toutes les inhibitions. L'espace est d'entrée de jeu coloré, euphorique, légèreté 
et indifférence, psychédélie douce ${ }^{42}$. » On voit très bien ce qui fait jouir la vidéo: les corps mangés par la lumière, dévorés par la fébrilité instable de l'électron. Les cellules de chair vidéographiées se font poreuses. Tout devient volatil, irradié. L'image est diaphane, fantômatique, spectrale. Mais ce qu'elle perd, cette image, comme lien avec le réel, elle le récupère en lien interstellaire, inter-angélique, inter-image.

Ce qui a changé, ce n'est pas seulement la texture de l'image, c'est aussi la caméra. Son errance joyeuse s'oppose au jeu très serré des champs/contrechamps de la narration cinématographique. Or, à qui appartient ce regardvidéo? J'ai déjà parlé des yeux de la foi, du regard du ressuscité. Mais ne serait-ce pas plutôt celui du vieux taxidermiste qui accepte la demande de M. Baadi, tout en cherchant néanmoins à le persuader de l'éternelle volupté d'exister? «Tu veux te passer du goût de la cerise?» Ce regard-vidéo, c'est le regard de personne et de tout le monde à la fois, mais c'est aussi, plus fondamentalement, et par sa défocalisation, un repos du regard. S'il faut que les figurants-soldats se reposent, comme Kiarostami les invite à le faire avec son porte-voix, il en va de même pour le regard. L'épreuve de la nuit aura été une épreuve du regard, comme si, ayant forcé les limites du visible, après une traversée du néant, le regard n'avait d'autre issue qu'un retour à l'évidence immédiate: tout recommence ici. Re-garder n’est-il pas un mouvement qui vise à reprendre sous garde ${ }^{43}$ ? En faisant ressurgir, voire renaître l'image sur un autre support, Kiarostami intensifie notre regard sur elle. Et cette intensification du regard conduirait à exacerber chez le spectateur le désir d'un monde luimême meilleur. Un monde où les soldats cueilleraient des fleurs... "Qui a passé ce seuil est libre de voir autrement le monde des apparences", écrit Ishagpour $^{44}$.

42. Pascal Bonitzer, Le champ aveugle. Essais sur le cinéma, Paris, Cahiers du cinéma, Éditions Gallimard, 1982, p. 41.

43. Jean Starobinski, L'œil vivant, Paris, Éditions Gallimard, coll. «Le Chemin», 1961, p. 11. Starobinski rappelle que la racine du mot regard «ne désigne pas primitivement l'acte de voir, mais plutôt l'attente, le souci, la garde, la sauvegarde, affectée de cette insistance qu'exprime le préfixe de redoublement ou de retournement» (p. 11). Le très beau texte de Jean-Luc Nancy sur le cinéma de Kiarostami, L'évidence du film, part de cette prémisse en mettant de l'avant le terme de "prégnance» du regard.

44. Youssef Ishagpour, Le réel, face ou pile, p. $9^{8 .}$ 
Apparences du monde et errance joyeuse d'une caméra captant tout ce qui lui tombe sous les yeux. Il n'y a pas de clôture de la représentation dans Le goût de la cerise, et sa deuxième fin vidéographique est bien plus de l'ordre d'un processus de capture d'intensités: celle du vent, de la lumière, de la musique. Le post-scriptum raconte-t-il encore? Je dirais que oui, dans la mesure où ce qui est mis de l'avant ici, c'est une autre puissance du récit cinématographique, une puissance qui ressortirait davantage à l'opsis qu'à la mimésis ${ }^{45}$. Cette autre puissance du récit cinématographique, c'est une puissance d'intensité sensible. Par rapport à la littérature, et au faible pouvoir sensible de son médium (langage, logos), le cinéma aurait ceci de spécifique: son dispositif matériel (l'enregistrement) lui donne d'emblée un accès immédiat au sensible.

Arrêtons-nous un instant sur la musique dans la mesure où le petit postscriptum est tout autant une image sonore qu'une image visuelle. Déjà l'image noire, qui était aveugle, n'était pas sourde. C'est par le son que nous quittions l'ancienne image de la vie pour entrer dans la nouvelle. La fin de la narration cinématographique s'éteignait lentement avec le bruit, de plus en plus assourdi, de la pluie qui tombe, et le début de la métanarration vidéographique s'ouvrait avec le bruit, de plus en plus fort - et jusqu'à ce que l'œil les perçoive dans l'autre image - de soldats en exercice et d'un sifflet qui en rythmait le pas de course. Le post-scriptum est sous l'emprise de la musique. Aucun critique ne s'est intéressé à cet aspect du film. On l'a souvent noté : la vidéo ne se regarde pas, elle s'écoute. Le regard vidéo est un regard qui, très souvent, passe par l'oreille avant de passer par l'œil. Dans ce petit post-scriptum, l'image obéit au son. Kiarostami lui-même est filmé avec son porte-voix qui avertit les figurantssoldats que le tournage est terminé et que l'équipe n'est là que pour la prise de son. Et c'est précisément à ce moment-là qu'une musique envahit l'image - sorte de marche funèbre style New Orleans - et qui est peut-être un des derniers traits d'ironie du film. Sur des images de prise de son en direct, une musique extradiégétique se déploie et termine le film jusqu’à la fin du générique. Seul le bruit du souffle du vent, lui-même fortement intensifié, lui fera concurrence. La vraie fin du film est musicale. Kiarostami termine son film par «l'esprit de la musique». Pas de dénouement ici, mais l'esprit de la musique.

45. Je reprends cette distinction à Jacques Rancière qui dans La fable cinématographique écrit: «C'est pourquoi l'art des images mobiles peut renverser la vieille hiérarchie aristotélicienne qui privilégiait le muthos - la rationalité de l'intrigue - et dévalorisait l'opsis - l'effet sensible du spectacle. (Paris, Éditions du Seuil, 2001, p. 8) 
Pas de «deus ex machina», mais la consolation métaphysique qui est, selon Nietzsche, dans l'esprit de la musique ${ }^{46}$. Si le post-scriptum nous enchante à ce point, ne serait-ce pas par cet aspect incantatoire, évanescent, dionysiaque? Ainsi, il n'y aurait rien à interpréter dans le post-scriptum car il serait lui-même une interprétation dans le sens d'exécution d'une musique, de la façon dont une œuvre est exécutée.

Rédemption, résurrection, sanctification, grâce, bénédiction, révélation, le vocabulaire des commentateurs pour décrire l'image du post-scriptum, a une connotation religieuse. Kiarostami, quant à lui, propose un terme plus neutre, celui d'accomplissement. Que tout puisse s'accomplir en un seul mouvement, comme par enchantement, est ce que me semble avoir voulu Kiarostami. Or, seul le cinéma peut permettre une telle expérience.

Je cite le passage:

Beaucoup de gens, à l'issue de la projection, se sont posés la question: "Pourquoi y a-t-il, à la fin, une scène de tournage du film? " C'est aussi une manière de dire que la vie continue à travers le cinéma...

Ce n'est pas là mon but. Quand le personnage descend dans la fosse, à la fin du film, pendant une minute, c'est le noir. Et la lune disparaît derrière les nuages, comme dans un fondu au noir. Ici, la vie, le cinéma et la lumière ne font plus qu'un. Sur l'écran, on ne voit plus rien. Puis, le premier signe de vie, six mois après, un petit matin de printemps, apparaît à l'image. Ainsi le noir de l'amorce est la matérialisation de la mort, du néant. Mais, le petit matin, la verdure - image de la vie - est aussi une image de cinéma. Quand la lumière disparaît, la lumière, la nature et la vie s'accomplissent en un seul mouvement. Même sans montrer la caméra, cela aurait eu le même sens: cette partie finale est une forme de résurrection; après l'obscurité totale, la vie nouvelle. (AK, p. 8o-81)

Le film aura donc été ce voyage initiatique allant vers l'accomplissement de l'image. «Dans toute la poésie mystique de l'Iran, il y a l'idée d'un voyage initiatique qui va vers l'accomplissement. » (AK, p. 82) On aurait ici une transposition cinématographique de cette grande tradition poétique à laquelle se réfère souvent Kiarostami.

Mais je reviens à la question de l'obscurité et de la lumière, sur laquelle insiste Kiarostami, et qui est au fondement du cinéma, de son expérience de

46. Friedrich Nietzsche, La naissance de la tragédie, trad. Geneviève Bianquis, Paris, Éditions Gallimard, coll. «NRF », 1985 [1872], p. 112. 
visionnement. «Au début, j’imaginais, nous dit Kiarostami, qu’on éteignait les lumières de la salle de cinéma pour mieux voir les images sur l'écran. J'ai regardé plus attentivement les spectateurs assis confortablement dans leur siège et j'ai constaté qu'il y avait une raison beaucoup plus importante: cette obscurité permettait à chaque spectateur de mieux se séparer des autres et d'être seul, d'être à la fois parmi les autres et d'être séparé d'eux. » (AK, p. 69) Ainsi nous serions ce regard de M. Baadi qui, du fond de sa sa tombe, est simultanément éclairé et obscurci par le jeu cosmique de la lune, des nuages et des éclairs. Et l'image noire deviendrait la salle de cinéma au moment que nous prisons le plus, qui est peut-être le moment de la plus grande jouissance-cinéma, je veux dire ce court laps de temps où l'obscurité se fait dans la salle, où les voix s'éteignent peu à peu, et où nous attendons que surgisse le point lumineux.

À un critique qui lui demande s'il n'allait pas tourner le dernier plan vidéo en film, Kiarostami répond que non, qu’il va le laisser tel quel, séparé du film. Ce petit post-scriptum nous dit le cinéaste

est comme un film d'amateur, un film familial, tourné en vidéo, dans une atmosphère d'intimité, comme si on filmait la vie, la résurrection. C'est un document que l'on a annexé au film. Je ne veux pas que cette partie soit partie intégrante du film. La vidéo lui donne un aspect documentaire, il n'y a pas de mise en scène. (AK, p. 85$)$

Kiarostami descend de son socle d'auteur consacré, et filme «comme tout le monde », en amateur, un film familial. La narration du post-scriptum met en place un autre affect que celui de la narration cinématographique, un affect de proximité avec le spectateur. Son immédiateté informelle, l'absence de fini ne lui donne-t-elle pas une allure de vie ordinaire, d'images de la vie qui appartiennent à tous parce que tous seraient susceptibles de les filmer? «L'insignifiance de la vie qui se tend ses images ${ }^{47}$.»

Une méditation philosophique peut-elle avoir une fin? Ou plutôt, comment la terminer, puisqu'il faut bien terminer? Je dirai, par la promesse d'une suite. La fin du film Le goût de la cerise ne serait-elle pas le début d'un autre film de Kiarostami? Le dernier plan du post-scriptum du Goût de la cerise et le premier plan du film qui lui succédera, Bad ma ra khahad bord (Le vent nous emportera, 1999), est celui d'une voiture qui s'éloigne dans les zigzags de la route. «Comme un résumé emblématique de ce cinéma écrit Nancy : le trajet 

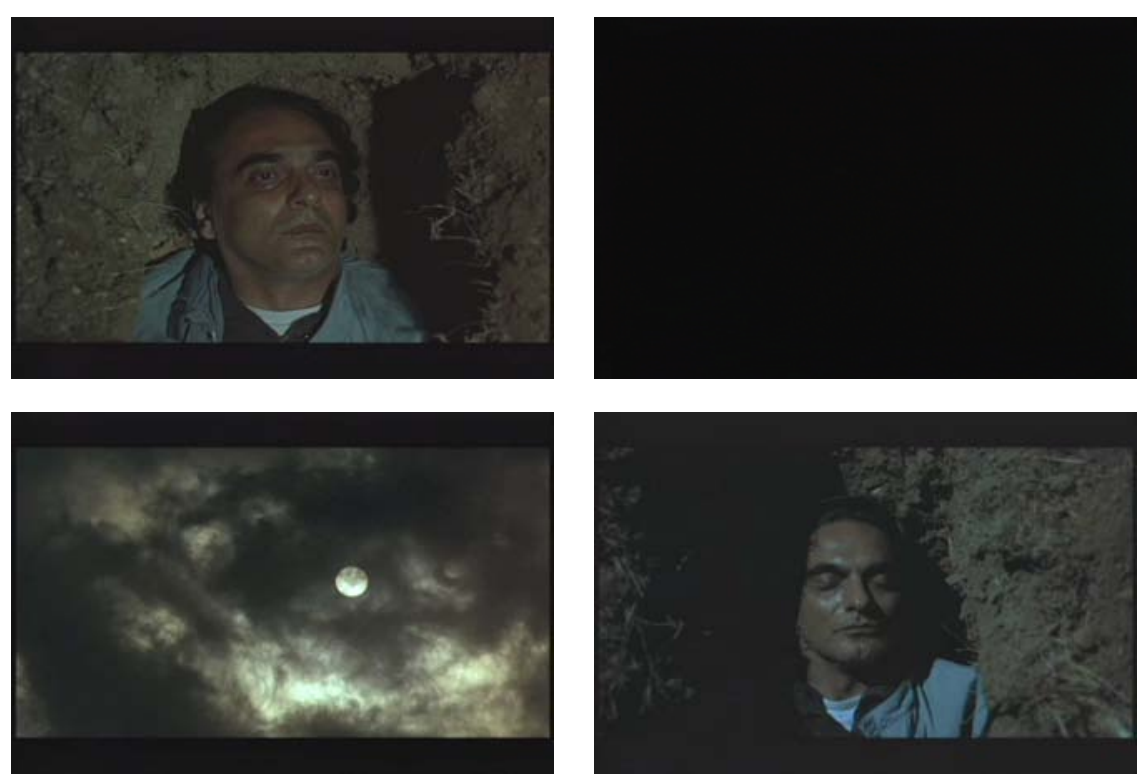

(C) Abbas Kiarostami, Le goût de la cerise, Copyright Marin Karmitz/Abbas Kiarostami, 1997 (photogrammes extraits du film).

en zigzag d'une personne ou d'une voiture sur fond d'un paysage immuable ${ }^{48}$. » Ishagpour souligne avec raison combien les films de Kiarostami semblent «sortir les uns des autres au sens d'une ouvre complète ${ }^{49}$ ». Nous sommes à la fin et pourtant, nous sommes au commencement. Mais il n'y a pas que ce motif récurrent de la voiture qui fait signe au film suivant, il y a aussi le vent. Le postscriptum ne nous dit-il pas, de façon visuelle et sonore que «Le vent nous emportera... »? Cet énoncé poétique est tiré d'un poème de Forough Farrokhzad. Selon Kiarostami,

[...] de son vivant, elle était très célèbre, et son regard est proche de celui de Khayyâm. Le poème de Forough qui est récité dans mon film est l'un des meilleurs, et le titre du film, Le vent nous emportera vient de ce poème: un jour ou l'autre, le vent nous emportera, comme une feuille $\operatorname{morte}^{50} \ldots$

48. Jean-Luc Nancy, L'évidence du film, p. 25.

49. Youssef Ishagpour, Le réel, face ou pile, p. 99.

50. Thierry Jousse et Serge Toubiana, «Un film n’a pas de passeport..., Entretien avec Abbas Kiarostami », p. 31. 

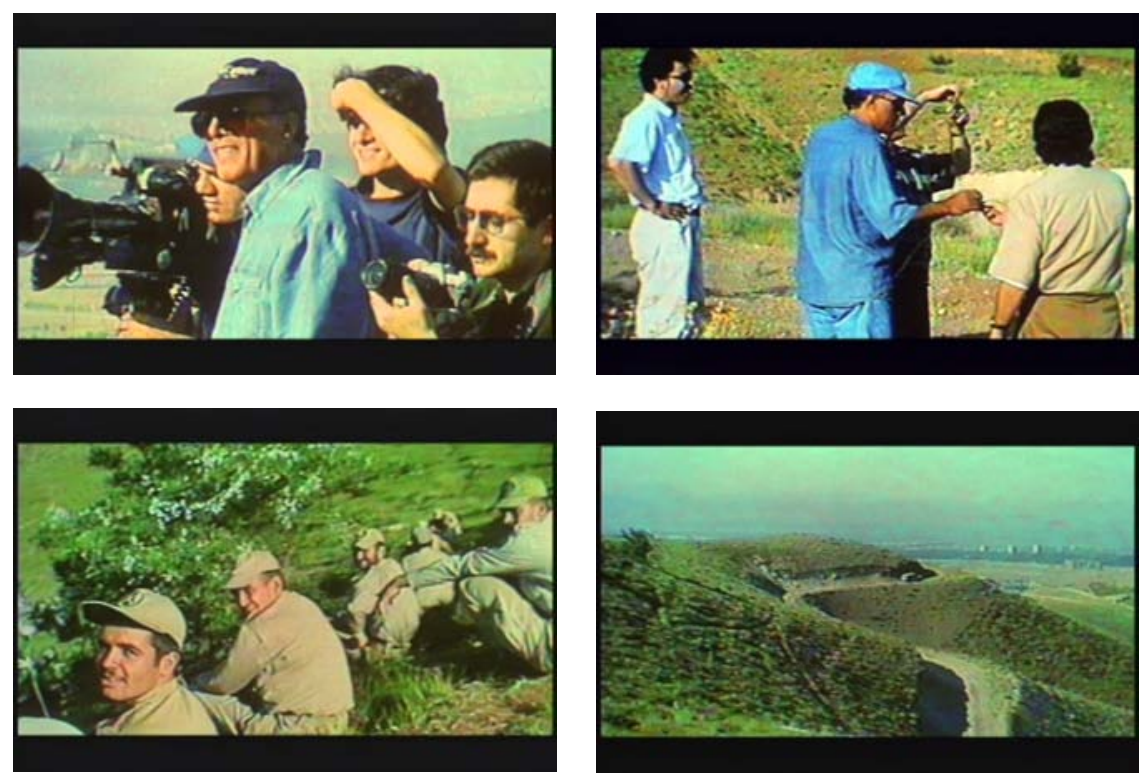

(C) Abbas Kiarostami, Le goût de la cerise, Copyright Marin Karmitz/Abbas Kiarostami, 1997 (photogrammes extraits du film).

On songe à Kierkegaard qui, à la fin de son volumineux ouvrage Les miettes philosophiques, promet une suite. Ce sera Post-scriptum aux Miettes philosophiques, ouvrage encore plus volumineux que le précédent. Il écrit:

Ainsi fut faite la promesse d'un suite. C'est pourquoi il est dans l'ordre qu'elle soit tenue dans un ouvrage subséquent, et l'auteur ne peut aucunement être accusé, si tant est qu'il y ait quelqu'un chose d'important dans toute l'affaire, d'avoir dit, à la manière des femmes, le plus important dans un post-scriptum ${ }^{51}$.

51. Søren Kierkegaard, Post-scriptum aux Miettes philosophiques, trad. Paul Petit, Paris, Éditions Gallimard, 1968 [1846]. 